\title{
Design of Controllers for Higher-Order-plus-Delay-Time Processes: A Practical Solution
}

\author{
Gajanan M. Malwatkar \\ Department of Instrumentation \& Control Engineering \\ Vishwakarma Institute of Technology \\ Pune-411037, India
}

\author{
Laxman M. Waghmare \\ Department of Instrumentation Engineering \\ SGGS Institute of Engineering \& Technology \\ Vishnupuri, Nanded-431606, India
}

\begin{abstract}
In this paper, a simple design method of proportional-integral (PI) controllers is proposed for higher order (HO)-plus delay time (HOPDT) processes. This controller is designed to handle higher order processes with long dead times, long time constants, and monotonic or oscillatory responses. The method is based on the real and imaginary values of the higher order processes for the desired settling time, and constraints on the complementary sensitivity function to handle the high frequency noise rejection. The procedure seems to be simpler, effective and improved performance can be expected of the various processes. The method has guarantee of existence of the solution. A simulation example and real time experimental level system are included to show the effectiveness, simplicity and practical applicability of the proposed method.
\end{abstract}

\section{Categories and Subject Descriptors}

J.7 [COMPUTERS IN OTHER SYSTEMS]: Industrial

Control, Process Control and real time

\section{General Terms}

Design, Theory, Experimentation.

\section{Keywords}

Delay time, Desired settling time, High order systems, Sensitivity function, PI controllers.

\section{INTRODUCTION}

Modern control theory has been developed significantly in the last two decades, however the use of the proportionalintegral(PI)-derivative (PID) controllers in various industries remained unaffected. The PID controllers are effectively used in many industries including process control, motor drives, magnetic and optic memories, automotive, flight control, instrumentation, chemical and petrochemical etc. The main reason of PID being used is its advantage of simple structure, good stability, high reliability, and simplicity of implementation in practice by control and process engineers. Over the past 60 years, the number of methods to design and tune the PID controller parameters have been developed for stable processes [1]-[3]. Some of them includes, the Coon-Cohen reaction curve, the Ziegler-Nichols step response, Ziegler-Nichols ultimate cycling, the Ziegler-Nichols frequency-response method, Internal model control, error-integral criteria (IAE, ISE, ITAE etc.) However, these tuning methods have certain limitations. They do not provide good tuning parameterizations for HO/HOPDT processes. The gain and phase margin (GPM) specifications methods have used in many applications to design the PID type controllers [4]-[7]. An important measures of robustness have been served by the GPM methods. The damping factor of the systems is related to phase margin and therefore served as a performance measure of the system. In GPM the solutions are normally obtained by numerically or graphically by means of trial-and-error, generally using Bode plots.

Many of the industrial processes have high order dynamics and includes small or large delay time, which makes difficulty in the design of low order feedback controllers. For such systems, the controller design methods like GPM and those incorporate small dynamic information of the systems have been failed. As a result the best methods of PI (PID) controllers design for higher order processes are always adopted time to time resulted from the research algorithms [8]-[9]. In control system design, the reduced model makes the synthesis, analysis and design of controller simpler. The reduction of high order processes to a reduced order processes have been a topic of interest of many researchers (e.g. [10]-[13]). The most of the prevalent controller tuning techniques for higher order processes are based on the reduced order models. The success of the controller depends on the accuracy of reduced order models. Any mis-match between plant and model may lead to serious problem and there is no guarantee of satisfactory performance of the process. It is true that the simple PI or PID controllers are normally preferred for high order and/or delay time linear-time-invariant systems. Therefore, many researchers and academicians follow the path of low order controllers design for HO/HOPDT processes.

In this paper, the higher order process dynamics at dominant poles are used to calculate the parameters of the PI (with negligible D action) controllers. The desired closed-loop settling time is used to calculate the complex term in the complex plane. The linear simultaneous equations are achieved from characteristics equations and complimentary sensitivity functions. The parameters of the controllers are computed by solving constrained linear equations. The stability of closed-loop systems is always guaranteed in an acceptable operating regions by the designed controller. The proposed method is verified on several simulation examples of HO/HOPDT, including those that can not be easily solved with an existing techniques. 


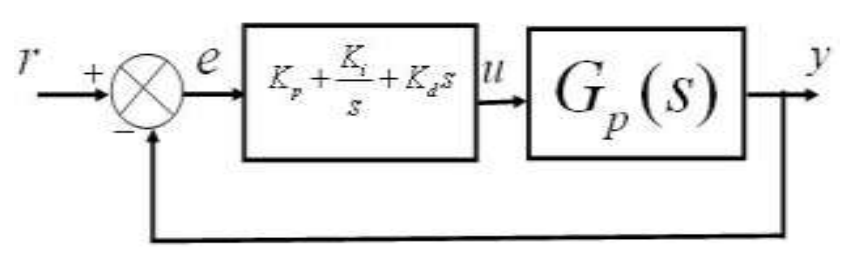

Fig. 1 Structure of PID with plants

A real time experiment is carried out on level control system and an effectiveness of proposed method is verified.

The organization of this paper is as follows. The proposed method is illustrated in section 2 while section 3 includes simulation examples. Section 4 is devoted to real time experimentation and the general conclusions are summarized in section 5 .

\section{THE PROPOSED METHOD}

The single loop PID controller configuration with process is as shown in figure 1. Suppose the open-loop transfer function (OLTF) of the higher order plant is

$$
G_{P}^{1}(s)=\frac{b_{0}^{1} s^{p}+b_{1}^{1} s^{p-1}+b_{2}^{1} s^{p-2}+\ldots+b_{p}^{1}}{s^{q}+a_{1}^{1} s^{q-1}+a_{2}^{1} s^{q-2}+\ldots+a_{q}^{1}} e^{-t} s,
$$

where, $\quad p \leq q, \quad b_{i}^{1} \quad(i=0,1,2, \cdots, p) \quad$ and $\quad a_{j}^{1}$ $(j=1,2, \cdots, q)$ are constant coefficients. The Pade approximation replaces delay time term $e^{-t d}$. The model given by equation (1) with Pade approximation for delay time can be written as

$$
G_{p}(s)=\frac{b_{1} s^{n-1} \pm b_{2} s^{n-2} \mp b_{3} s^{n-3} \pm \ldots \pm b_{n}}{a_{0} s^{n}+a_{1} s^{n-1}+a_{2} s^{n-2}+\ldots+a_{n}},
$$

where $b_{i}(i=1,2, \cdots, n)$ and $a_{j}(j=0,1,2, \cdots, n)$ are constant coefficients. An exact analytical description of closedloop settling time and maximum peak overshoot is difficult to obtain for higher order systems. However, the relationship of second order system can be approximated to measure the performance of the systems. In the desired closed-loop response the maximum peak overshoot to be less than $10 \%$ and settling time to be longer than $10 s$ are assumed to ensure the location of the complex poles close to imaginary axis. The location of dominant poles in the complex plane can be computed as

$$
s=-a \pm j b \cong-\frac{4}{t_{s}}\left(1 \mp j \frac{\pi}{\sqrt{(\ln (0.1))^{2}}}\right)
$$

where, $a$ and $b$ are constant coefficients, and $l n$ represent natural logarithm.

The standard form of the PID controller is
$G_{c}(s)=K_{p}+\frac{K_{i}}{s}+K_{d} s=\frac{K_{d} s^{2}+K_{p} s+K_{i}}{s}$,

where, $K_{p}, K_{i}$ and $K_{d}$ represents proportional, integral and derivative gain respectively. The characteristic equation of the system with controller $G_{c}(s)$ for the structure as shown in Fig. 1 is

$1+G_{p}(s) G_{c}(s)=0$.

Suppose that the requirements of the closed-loop control performance in frequency or time domain are converted into a pair of conjugate poles [1] as $s_{d_{1,2}}=-a \pm j b$. The problem of the dominant pole placement is to find the PID parameters such that the closed-loop poles $s_{d_{1,2}}=-a \pm j b$ should be lie on the locus of $G_{p} G_{c}$ in the desired region. Hence the desired closed-loop response can be achieved by adjusting the controller parameters at $s=-a+j b$ or $s=-a-j b$. Substituting $s=-a+j b$ in equation (5)

$$
1+G_{c}(-a+j b)\left(X_{1}+j X_{2}\right)=0
$$

or

$$
\begin{aligned}
& {\left[K_{d}\left(a^{2}-b^{2}\right)-a K_{p}+K_{i}-j 2 a b K_{d}+j b K_{p}\right]} \\
& \left(X_{1}+j X_{2}\right)=a-j b,
\end{aligned}
$$

Where the terms in equation (6) are $X_{1}=\operatorname{Re}\left[G_{p}(-a+j b)\right]$ $X_{2}=\operatorname{Im}\left[G_{p}(-a+j b)\right] . R e$ and $\operatorname{Im}$ denotes real and imaginary term. After simplification, and comparisons of realreal and imaginary-imaginary parts in equation (6), it can be written as

$$
\begin{aligned}
& {\left[\left(a^{2}-b^{2}\right) X_{1}+2 a b X_{2}\right] K_{d}-\left[a X_{1}+b X_{2}\right] K_{p}} \\
& +X_{1} K_{i}=a
\end{aligned}
$$

and

$$
\begin{aligned}
& {\left[\left(a^{2}-b^{2}\right) X_{2}-2 a b X_{1}\right] K_{d}+\left[b X_{1}-a X_{2}\right] K_{p}} \\
& +X_{2} K_{i}=-b .
\end{aligned}
$$

The pole placements with controller can be ensured by verifying the magnitude and angle condition at $s_{d_{1,2}}$. For the structure as shown in Fig. 1, the magnitude condition at $s=-a+j b$ is

$$
\left|G_{p}(-a+j b) G_{c}(-a+j b)\right|=1
$$

or 
$\left|\left[\left(a^{2}-b^{2}\right) K_{d}-a K_{p}+K_{i}+j\left(b K_{p}-2 a b K_{d}\right)\right]\left[X_{1}+X_{2}\right]\right|$ $=|-a+j b|$

After simplification, equation (9) yields,

$K_{1} K_{d}^{2}+K_{2} K_{p}^{2}+K_{3} K_{i}^{2}+K_{4} K_{d} K_{p}+K_{5} K_{p} K_{i}+K_{6} K_{d} K_{i}=a^{2}+b^{2}$,

where,

$K_{1}=\left[\left(a^{2}-b^{2}\right) X_{1}+2 a b X_{2}\right]^{2}+\left[\left(a^{2}-b^{2}\right) X_{2}-2 a b X_{1}\right]^{2}$

$K_{2}=\left(a X_{1}+b X_{2}\right)^{2}+\left(b X_{1}-a X_{2}\right)^{2}$

$K_{3}=X_{1}^{2}+X_{2}^{2}$

$K_{4}=2\left[\left(a^{2}-b^{2}\right) X_{2}-2 a b X_{1}\right]\left(b X_{1}-a X_{2}\right)$

$-2\left[\left(a^{2}-b^{2}\right) X_{1}+2 a b X_{2}\right]\left(a X_{1}+b X_{2}\right)$

$K_{5}=2\left[\left(b X_{1}-a X_{2}\right) X_{2}-\left(a X_{1}+b X_{2}\right) X_{1}\right]$

$K_{6}=2\left[\left(a^{2}-b^{2}\right) X_{2}-2 a b X_{1}\right] X_{2}+2\left[\left(a^{2}-b^{2}\right) X_{1}\right.$

$\left.+2 a b X_{2}\right] X_{1}$

The angle condition for the system with controller at $s=-a+j b$ is,

$\angle\left[G_{p}(-a+j b) G_{c}(-a+j b)\right]= \pm 180^{\operatorname{deg}}(2 r+1) \bmod \quad r=0,1,2, \ldots$

or

$\tan ^{-1}\left(\frac{b K_{p}-2 a b K_{d}}{\left(a^{2}-b^{2}\right) K_{d}-a K_{p}+K_{i}}\right)+\tan ^{-1}\left(\frac{X_{2}}{X_{1}}\right)$

$-\tan ^{-1}\left(\frac{b}{-a}\right)= \pm 180^{\operatorname{deg}}(2 r+1)$.

After simplification, equation (11) can be wriiten as,

$$
\begin{aligned}
& {\left[a\left(a^{2}-b^{2}\right) X_{2}-2 a^{2} b X_{1}-2 a b^{2} X_{2}+b\left(a^{2}-b^{2}\right) X_{1}\right] K_{d}} \\
& +\left[-a^{2}-b^{2}\right] X_{2} K_{p}+\left[a X_{2}+b X_{1}\right] K_{i}=0
\end{aligned}
$$

To ensure a high frequency noise rejection a constraint on the complementary sensitivity function $\mathrm{S}$ can be defined as

$$
\begin{aligned}
& |S(-\alpha+j \beta)|=\left|\frac{G_{p}(-\alpha+j \beta) G_{c}(-\alpha+j \beta)}{1+G_{p}(-\alpha+j \beta) G_{c}(-\alpha+j \beta)}\right|_{d B} \leq A \quad d B \\
& \forall \omega \geq \omega_{s} \Rightarrow|S(-\alpha+j \beta)|_{d B}=A,
\end{aligned}
$$

or

$$
\begin{aligned}
& \frac{G_{p}(-\alpha+j \beta) G_{c}(-\alpha+j \beta)}{1+G_{p}(-\alpha+j \beta) G_{c}(-\alpha+j \beta)} \leq-\alpha+j \beta \\
& \forall \omega \geq \omega_{s} \Rightarrow|S(-\alpha+j \beta)|=\sqrt{\alpha^{2}+\beta^{2}},
\end{aligned}
$$

where, $\mathrm{A}$ is the desired value of the complementary sensitivity function for the desired frequency range $\omega_{s} \leq \omega \mathrm{rad} / \mathrm{s}$, and $\alpha$ and $\beta$ are real coefficients. A factor of $\left[1+G_{p}(s) G_{c}(s)\right]$ is much greater than unity in most of the practical cases over the frequency ( at $s=-\alpha+j \beta$ ) of interest. Thus the value of complimentary sensitivity function $A$ is always positive and less than unity. With $\alpha=a$ and $\beta=b$, the desired complementary sensitivity function is $A=\sqrt{a^{2}+b^{2}}$. The coefficients in sensitivity function are computed at $s=-\alpha+j \beta$. The desired sensitivity function in the range form $-5 d B$ to $-20 d B$ is assumed, but one can have a freedom to select any appropriate value of dominant poles such that the desired sensitivity function $A<0 \quad d B$. After simplification, with real-real and imaginary-imaginary comparison,

$$
\begin{aligned}
& Y_{11} K_{d}+Y_{12} K_{p}+Y_{13} K_{i}=a^{2}-b^{2} ; \\
& Y_{21} K_{d}+Y_{22} K_{p}=-2 a b+Y_{23} K_{i}
\end{aligned}
$$

where,

$$
\begin{aligned}
& Y_{11}=\left[X_{1}\left(a^{2}-b^{2}\right)+X_{2} a b+a X_{1}\left(a^{2}-b^{2}\right)+\right. \\
& \left.X_{2} 2 a^{2} b-2 a b^{2} X_{1}+b X_{2}\left(a^{2}-b^{2}\right)\right] \\
& Y_{12}=\left[-a X_{1}-b X_{2}-a X_{1} a-a X_{2} b+b^{2} X_{1}-b X_{2} a\right], \\
& Y_{13}=\left[X_{1}+a X_{1}+b X_{2}\right], \\
& Y_{21}=\left[X_{2}\left(a^{2}-b^{2}\right)-2 a b X_{1}-b X_{1}\left(a^{2}-b^{2}\right)\right. \\
& \left.-2 a b^{2} X_{2}-2 a^{2} b X_{1}+\left(a^{2}-b^{2}\right) a X_{1}\right], \\
& Y_{22}=\left[b X_{1}-a X_{2}+a X_{1} b+b X_{1} a+b^{2} X_{2}-a^{2} X_{2}\right],
\end{aligned}
$$$$
\text { and } Y_{23}=\left[X_{2}-b X_{1}+a X_{2}\right] \text {. }
$$

The design of controller is based on the equations (7), (8), (10), (12) (14) and (15), at least three equations should be fulfilled, since it has three parameters to tune. In this paper, the condition given in Eq. (14) is taken as the main function to minimize, and the conditions given in equations (7), (8), (12) and (15) are taken as equality constrains for the minimization. The optimization toolbox of Math-works MATLAB has been used to reach out the best solution with the minimum error. The function used for this purpose is called LINPROG, which finds the constrained minimum of a function of several variables. It solves problems of the form:

$$
\min _{x} f^{T} x
$$


such that $A x \leq b, A_{e q} x=b_{e q}$ and $l b \leq x \leq u b$, where $f$ is the function to be minimize, $A x$ and $b$ are matrices of inequalities constraints, $A_{e q}$ and $b_{e q}$ matrices of equality constraints, $l b$ and $u b$ defines a set of lower and upper bounds on the design variables, $x$, so that the solution is always in the range $l b \leq x \leq u b$. To ensure the positiveness values of controller parameters, nonlinearity constraints such as $K_{d}>0$, $K_{p}>0$ and $K_{i}>0$ are also included. The parameters of the controllers can be accepted only if the conditions given in equations (7), (8) (10) and (12) are fulfilled and a function given in equation (14) is with minimum value. It should be note that the constraint given in eq. (10) is not used in the LINPROG function, but it should be satisfy by the obtained controller parameters. This ensures the guaranteed dominant of the assigned poles in the locus of $G_{p} G_{c}(s)$.

\section{Design procedure:}

1. Find the dominant poles using the relation given by equation (3) such that $A d B \in(-5,-20) d B$.

2. Obtain the values of $X_{1}$ and $X_{2}$ at $s=-a+j b$.

3. Minimize the function given by eq. (14) with equality constraints of eqs. (7), (8), (12) and (15). Set $A=[$ ], $b=\left[\right.$ ], the initial starting point $x_{0}=$ [ ], $u b=$ [ ] and $l b=[$ ] in MATLAB's LINPROG function. Also apply the inequality constraints such as $K_{d}>0$, $K_{p}>0$ and $K_{i}>0$.

4. If the constraints given in equations (7), (8), (10), (12) and (15) are not satisfied, repeat the step 1 to 3 by changing the $t_{s}$. This ensures the robustness and guaranteed solution of the problem.

5. Finally, obtain the controller parameters $K_{d}, K_{p}$ and $K_{i}$ using the minimized variable $x$.

The proposed approach has superior features, including: simplicity; easy implementation; and good computational efficiency. Fast tuning of PID controller parameters yields highquality results.

\section{SIMULATION EXAMPLE}

A higher order with delay time process is simulated in MATLAB 7.0.1 to show effectiveness of the proposed method. The controller designed by proposed algorithm are compared with prevalent tuning techniques such as Wang et al.'s method [8], Ho et al.'s method [6] and latest method of Q.-G. Wang et al.'s [14] and Malwatkar et al.'s [9]. Wang et al.'s controller design method is based on the fitting of the process frequency response to a particular second-order plus dead time structure and Ho et al.'s method is based on gain margin and phase margin specifications which are set at 3 and 60 degree respectively. Q.-G. Wang et al's method is based on Nyquiest plot and root-locus for systems with and without delay time respectively. Malwatkar et al's method uses reduction of higher order system by frequency response data and design of controllers has been made for reduced order. These methods are the most suitable candidates for comparison since these are the best and latest methods compared with most of other tuning formulas.

Consider the process given in [8] with OLTF

$$
G_{P}(s)=\frac{1}{(s+3)^{5}} e^{-2 s} .
$$

The range of settling time $t_{s}$ for the desired closed-loop response can be approximated using the equation (3). Suppose the settling time to be no longer than 12 The values of complex term for this specifications are $s=-0.3333 \pm j 0.4548$. The real and imaginary components of $G_{p}(-0.3333+j 0.4548) \quad$ are $\quad X_{1}=-0.0025 \quad$ and $X_{2}=-0.0132$ respectively. The function

$$
f(K)=-0.0041 K_{d}+0.0106 K_{p}-0.0024 K_{i}
$$

given by eq. (14) is minimized by equality constraints

$$
\begin{aligned}
& -0.0026 K_{d}+0.0042 K_{p}-0.0055 K_{i}=0 \\
& -0.0038 K_{d}+0.0068 K_{p}-0.0025 K_{i}=0.3333 \\
& 0.0020 K_{d}+0.0033 K_{p}-0.0132 K_{i}=-0.4548 \\
& 0.0041 K_{d}+0.0013 K_{p}-0.0165 K_{i}=-0.3032 \\
& K_{d}>0, K_{p}>0, K_{i}>0,
\end{aligned}
$$

resulted from the equations (7), (8), (12) and (15). The resulting controller is,

$$
G_{c}(s)=67.16+\frac{51.12}{s} .
$$

The parameters of the controller satisfy all the constraints. Wang et al.'s, Ho et al.'s Malwatkar et al.'s methods give the controllers,

$$
\begin{aligned}
G_{c \text { Wang }}(s) & =58.62+\frac{49.78}{s}+22.09 s, \\
G_{c H o}(s) & =53.08+\frac{48.83}{s}+14.43 s
\end{aligned}
$$

and

$$
G_{c M}(s)=60.43+\frac{50.33}{s}+23.74 s
$$

respectively. By Q.-G. Wang et al.'s method, the range of proportional gain is $K_{p} \in(0,310)$ for the desired closed-loop response as stated above. Let $K_{p}=60$ to ensure the 


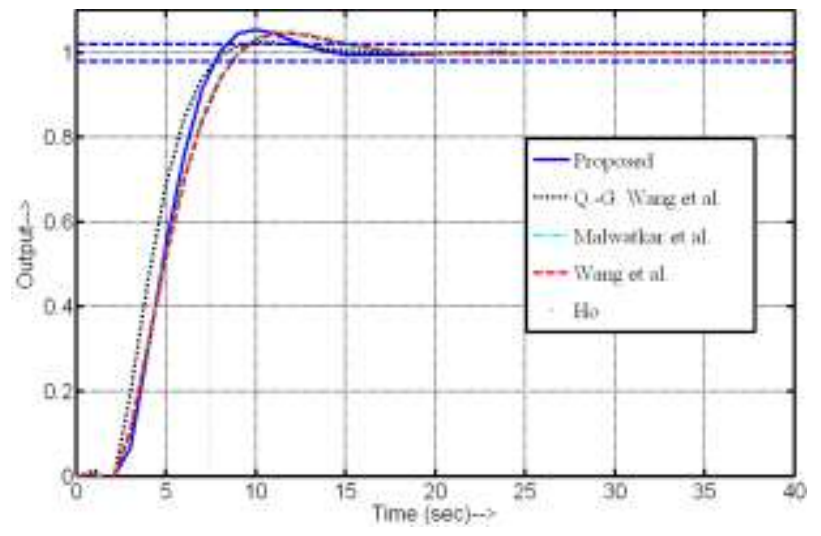

Fig. 2 Closed-loop responses with controllers (simulation example)

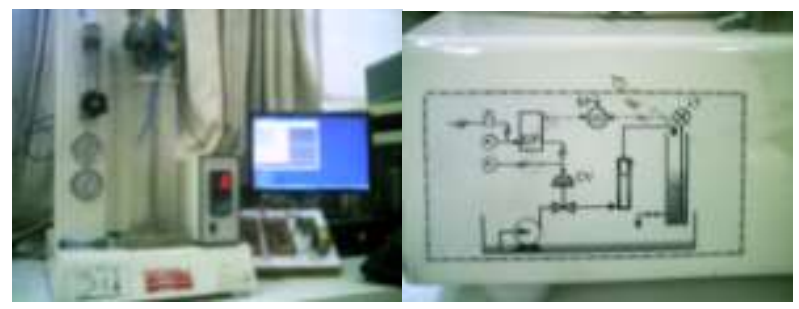

Fig. 3: Experimental Set-up and its portrayal. (LT: Level transmitter, E/P: Current to pressure converter, CV: Control Valve, SP: Set point, LIC: Level indicating controller)

positiveness of the controller parameters, the structure of the controller is,

$$
G_{c Q-G}(s)=60+\frac{46.32}{s}+2.465 s .
$$

The closed loop response of the process with all prevalent controllers and proposed controller is as shown in Fig. 2 to unit set point change at time $t=0$. The responses due to proposed controller is fast and the settling time is less than $12 \mathrm{~s}$ with 5 $\%$ overshoot. The responses of Wang et al.'s, Ho et al.'s and Malwatkar et al.'s controllers are identical with large settling time as compared to proposed. The Q.-G. Wang et al.'s method gives less overshoot and settling time is approximately equal to proposed method.

\section{Real Time Implementation}

The applicability and effectiveness of the proposed method has been carried on real-time level control experiment. The set-up of level loop and its representation is shown in Fig. 3. The system mainly includes gravity flow tank, pneumatic valve, capacitive type sensor and transmitter, electro-pneumatic converter, all connected in series. Since all the elements in this system are first order with small time constant, it is a type zero and conditionally stable system. The system is self-regulatory and the model obtained for this system is linearized version around an operating point. Gravity flow tank is a first order system with a time constant higher than the time constants of other elements in the set-up (pneumatic valve, transmitter, electro-pneumatic converter

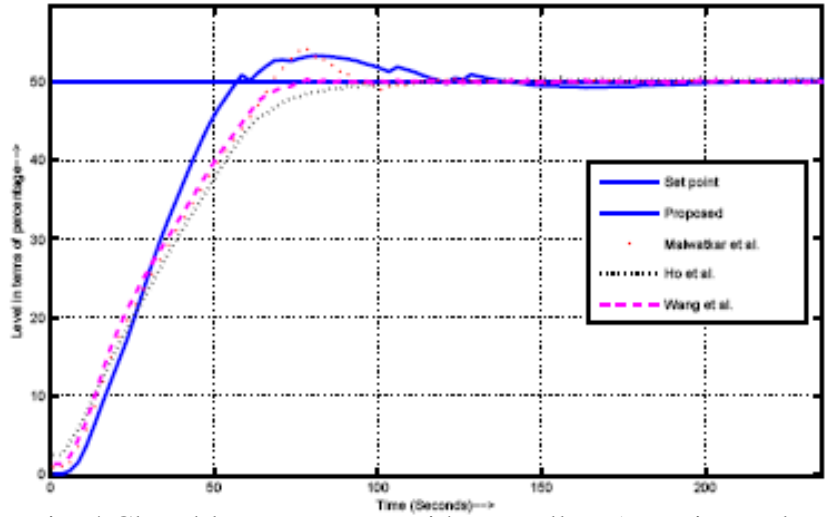

Fig. 4 Closed-loop responses with controllers (experimental results)

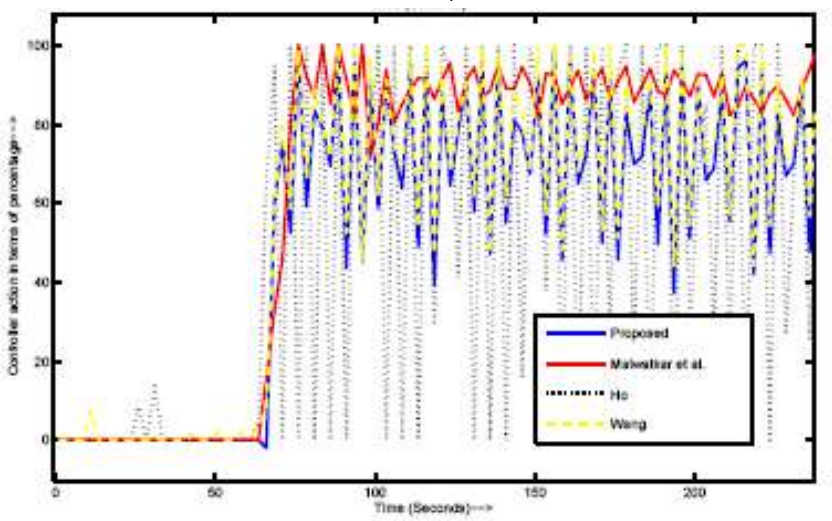

Fig. 5 Controller actions (current from PC to E/P converter)

etc.). The open loop identification carried out by designing appropriate pseudo random binary signal (PRBS) signal. The system connected to computer through a data acquisition card. To find an empirical model of the system, the open loop output data collected by exciting the system with PRBS from stored input vector (computer). The PRBS which is voltage signal converted into pressure signal through voltage to current converter, current to pressure converters and applied to the pneumatic valve as input signal to the system. Effect of the PRBS signal on water level is recorded using capacitive level sensor and transmitter, which is a current signal. The level in terms of current signal converted into voltage signal and stored as output vector. The model of the level system together with components such as valve, I/P converter, level sensor and transmitter identified by using input-output data . Fitting of input-output data has been made using autoregressive with external input model with orders of [ $\left.\begin{array}{lll}5 & 1 & 0\end{array}\right]$, that is five poles, no zeros and zero delay. The discrete time model converted into continuous time using matched polezero method. For the model obtained thus, the controller parameters are obtained by the proposed method and the performance of the overall closed loop system is checked.

The identified continuous time system with transfer function is,

$$
G_{P}(s)=\frac{1.62}{p_{1} p_{2} p_{3}} 10^{-6},
$$


where,

$p_{1}=s+0.05055, \quad p_{2}=s^{2}+0.07171 s+0.164$ and

$p_{3}=s^{2}+0.1901 s+0.03405$. The controller for this system is ,

$$
G_{c}(s)=47.27+\frac{7.179}{s} .
$$

The controllers given by Ho et al. and Wang et al. are

$$
G_{c H O}(s)=106.3+\frac{6.719}{s}+421 s
$$

and

$$
G_{c \text { Wang }}(s)=79+\frac{9.041}{s}+177 s
$$

respectively.

The PID parameters given by Wang et al. and Ho et al. strategies together with tuned parameter given by proposed method are used to check the overall performance of the system. The Step change of set point from empty tank to 50 percentage is applied at time $t \geq 0$. The closed loop response of systems with controllers are as shown in Fig. 4 and the output of the controller in terms of percentage closing of the valve is shown in Fig. 5. The response given by proposed controller is faster than the Wang's and Ho's controller. Q.-G. Wang et al.'s method have no solution for this system. The proposed controller gives overshoot as compared to others but the controller action is smooth. The Wang's and Ho's controller gives more oscillatory action of the control valves (or controller output) as shown in Fig. 5. Such natures of controller action consumes more power and create the bumping of the valves.

\section{Conclusions}

A simple method of controller design for higher order with delay time processes has been proposed. The method is demonstrated through a simulation example and a real time experimentation. The design method is not based on reduced order models and hence enhanced performance of the processes can be expected. Once the location of dominant poles obtained, simple and effective design rules presented in the paper are used to control the any process with different characteristics. Since the proposed design method incorporates pole placement by PI(D) controllers, the closed-loop process with designed PI controller results in good set point responses. By the designed controller, the closed loop response would be fast or slow which is depend on the desired settling time. The output response of the processes by proposed controller is always smooth and bump-less action of the controller can be expected. The proposed method would be extended to the non-minimum phase and unstable higher order systems.

\section{ACKNOWLEDGMENTS}

The authors would like to thank University of Pune, Pune-India for the research funding vide letter no. BCUD/14 dated 10/04/2008. The authors are thankful to Vishwakarma Institute of Technology, Pune for providing experimental set-up and related facilities at Process Instrumentation and Control laboratory.

\section{REFERENCES}

[1] Astrom, K. J., and Hagglund T, 1995. PID controllers: theory, design and tuning, USA: Instrument Society of America.

[2] Astrom, K. J., Hagglund, T., Hang, C. C., and Ho, W. K. , 1993. Automatic Tuning and Adaptation for PID Controllers-A Survey, IFAC J. Control Eng. Practice, Vol.1, no.4, pp.699-714.

[3] Astrom , K. J., and Hagglund, T., 2001. The Future of PID Control, IFAC J. Control Engineering Practice, Vol. 9, pp. 1163-1175.

[4] Ching-Hung Lee, 2004. A Survey of PID Controller Design Based on Gain and Phase Margins (Invited Paper), International Journal of Computational Cognition Vol. 2, No. 3, pp. 63-100.

[5] H. W. Fung, Q. G. Wang, and T. H. Lee, 1998. PI tuning in Terms of Gain and Phase Margins, Automatica, vol. 34, No. 9, pp. 1145-1149.

[6] W. K. Ho, C. C. Hang, and L. S. Cao, 1995. Tuning of PID controllers based on gain and phase margin specifications, Automatica, vol. 31, no. 3, 497-502.

[7] W. K. Ho, O. P. Gan, E. B. Tay, and E. L. Ang, 1996. Performance and Gain and Phase Margins of Well-known PID Tuning Formulas, IEEE Trans. Control Systems Technology, Vol. 4, No. 11, pp. 473-477.

[8] Qing-Guo Wang, Tong-Heng Lee, Ho-Wang Fung, Qiang $\mathrm{Bi}$, and Yu Zhang, 1999. PID Tuning for Improved Performance, IEEE Transactions on Control System Technology, Vol. 7, No. 4, 457-465.

[9] G. M. Malwatkar, S. H. Sonawane and L. M. Waghmare, 2009. Tuning PID controllers for higher-order oscillatory systems with improved performance, ISA Transactions, 48, 347-353

[10] A. Lepschy, and U. Viaro,1983. A note on the model reduction problem, IEEE Transactions on Automatic Control, vol. AC-28, no. 4, 525-527.

[11] W. Krajewski, A. Lepschy, and U. Viaro, 1994. Reduction of linear continuous-time multivariable systems by matching first and second-order information, IEEE Transactions on Automatic Control, vol. 39, no. 10, 2126-2129.

[12] N. K. Sinha, 1992. Reduced order models for linear systems, IEEE International Conference on Systems, Man and Cybernetics, 1992.

[13] P. S. Shingare, 2007. Fixed and Interval Model Reduction Techniques for Control System Design, Ph. D. thesis, Indian Institute of Technology Bombay, Mumbai.

[14] Qing-Guo Wang, Zhiping Zhang, Karl Johan Astrom, and Lee See Chek, 2009. Guaranteed dominant pole placement with PID controllers, Journal of Process Control 19, 349352. 